Published in final edited form as:

Phys Chem Chem Phys. 2016 July 27; 18(30): 20199-20207. doi:10.1039/c6cp00632a.

\title{
THz time-domain spectroscopy of mixed $\mathrm{CO}_{2}-\mathrm{CH}_{3} \mathrm{OH}$ interstellar ice analogs $†$
}

\author{
Brett A. McGuire ${ }^{a, b, \ddagger}$, Sergio loppolo ${ }^{c, d}$, Marco A. Allodi ${ }^{b, e}$, Geoffrey A. Blake $^{b, d}$ \\ aNational Radio Astronomy Observatory, 520 Edgemont Rd, Charlottesville, VA, 22903, USA. \\ bDivision of Chemistry and Chemical Engineering, California Institute of Technology, Pasadena, \\ CA, 91125, USA \\ 'Department of Physical Sciences, The Open University, Walton Hall, Milton Keynes, MK7 6AA, \\ UK \\ ${ }^{\mathrm{d} D i v i s i o n}$ of Geological and Planetary Sciences, California Institute of Technology, Pasadena, CA, \\ 91125, USA. \\ eDepartment of Chemistry, The Institute for Biophysical Dynamics, and the James Franck \\ Institute, The University of Chicago, Chicago, IL, 60637, USA
}

\section{Abstract}

The icy mantles of interstellar dust grains are the birthplaces of the primordial prebiotic molecular inventory that may eventually seed nascent solar systems and the planets and planetesimals that form therein. Here, we present a study of two of the most abundant species in these ices after water: carbon dioxide $\left(\mathrm{CO}_{2}\right)$ and methanol $\left(\mathrm{CH}_{3} \mathrm{OH}\right)$, using TeraHertz $(\mathrm{THz})$ time-domain spectroscopy and mid-infrared spectroscopy. We study pure and mixed-ices of these species, and demonstrate the power of the $\mathrm{THz}$ region of the spectrum to elucidate the long-range structure (i.e. crystalline versus amorphous) of the ice, the degree of segregation of these species within the ice, and the thermal history of the species within the ice. Finally, we comment on the utility of the THz transitions arising from these ices for use in astronomical observations of interstellar ices.

\section{Introduction}

Cometary bombardment and meteoritic impacts have long been known to deliver substantial quantities of water and organic molecules to Earth, which may well have been the primordial prebiotic seeds of life. ${ }^{1}$ This raises the question: What is the ultimate origin of this material? While some chemical evolution can certainly occur in situ in these icy bodies, a substantial portion of the molecular material is inherited directly from the parent molecular cloud. ${ }^{2}$ Thus, a thorough understanding of the primordial origins of our prebiotic molecular reservoir necessitates an examination of the genesis of this material in star- and planetforming interstellar clouds. ${ }^{3}$

\footnotetext{
$\dagger$ Electronic supplementary information (ESI) available. See DOI: 10.1039/c6cp00632a bmcguire@nrao.edu.

$\ddagger_{B}$. A. M. is a Jansky Fellow of the National Radio Astronomy Observatory.
} 
Generally, simple, unsaturated molecules, as well as a number of long-chain hydrocarbons and fullerene species, can efficiently form via gas-phase ion-molecule reactions in the interstellar medium (ISM). ${ }^{4}$ There is strong evidence, however, that more complex hydrogenated species, up to and including amino acids, are formed almost exclusively via reactions on and within the icy surface of interstellar dust grains. ${ }^{4-10}$ For example, the presence and abundance of methyl formate, one of the most prevalent interstellar complex organic molecules, has been argued to be explainable only through formation via radicalradical recombination reactions in these icy bodies. ${ }^{11}$ Indeed, a recent laboratory study has shown that three abundant complex molecules - methyl formate, glycolaldehyde, and ethylene glycol - are efficiently formed in the solid phase through recombination of free radicals formed via $\mathrm{H}$-atom addition and abstraction reactions that occur during the hydrogenation of $\mathrm{CO}$ ice at $15 \mathrm{~K}$ under dense molecular cloud conditions. ${ }^{12}$

Despite their origins in molecular ices, the most complex molecule yet detected in the condensed-phase of the ISM is $\mathrm{CH}_{3} \mathrm{OH} .{ }^{13}$ Indeed, only six species $-\mathrm{H}_{2} \mathrm{O}, \mathrm{CO}, \mathrm{CO}_{2}$, $\mathrm{CH}_{3} \mathrm{OH}, \mathrm{NH}_{3}$, and $\mathrm{CH}_{4}$ - have been securely identified observationally, although there is strong evidence for the additional presence of $\mathrm{H}_{2} \mathrm{CO}, \mathrm{OCN}^{-}$, and OCS. ${ }^{13}$ Thus, while characterizing these ices is critical for understanding the genesis of complex prebiotic material, we are currently limited in our ability to constrain models of chemical evolution in these condensed-phase environments where it occurs.

Much attention in the laboratory has been focused on the formation, destruction, and reaction of species within interstellar ice analogs, primarily using mid-infrared (mid-IR) spectroscopy. ${ }^{14}$ These studies, while crucial, have difficulty unambiguously measuring a critical component of the equation: the physical structure of the ice, which can have profound effects on reactions within the bulk material. ${ }^{5}$ Indeed, mid-IR spectroscopy is not the most powerful tool for examining this long-range structure, as, in general, the signals observed in the mid-IR only probe intra-molecular modes which are characteristically perturbed by the surrounding ice structure. In the far-IR, or TeraHertz (THz, 0.1-10 THz, $30-3000 \mu \mathrm{m})$, region of the spectrum, however, it is the softest degrees of freedom of the ice (i.e. inter-molecular modes) that are probed. ${ }^{15}$

These inter-molecular modes offer a unique probe of ice structure (i.e. crystalline vs. amorphous ice). The thermal history of the ice is revealed as well, since the change in ice from amorphous to crystalline phases is a non-reversible process that starts at $\sim 110 \mathrm{~K}$ for water ice under laboratory (ultra) high vacuum conditions. ${ }^{16-21}$ Recent observations of crystalline water ice have suggested this may be a powerful tool in studies of the evolution of planetary systems from the initial collapse phase through planet formation. ${ }^{22}$ The extreme sensitivity of the $\mathrm{THz}$ region to these structural modes opens the door to the study of species less-abundant than water, that are just as critical to our understanding of both physical and chemical evolution within forming systems. The Far Infrared Field-Imaging Line Spectrometer (FIFI-LS) aboard the Stratospheric Observatory for Infrared Astronomy (SOFIA) offers bandwidth that is well-matched to these THz modes, covering 51-203 $\mu \mathrm{m}$ (1.5-5.9 THz) across two spectral bands. 
The $\mathrm{THz}$ region of the spectrum has historically been challenging to access. Recent advances in generation and detection techniques for THz photons, however, have allowed us to construct a broadband, sensitive, and coherent spectrometer whose spectral resolution is ideally-matched to the modes arising from the bulk motion of interstellar ice analogs. We have previously reported on THz time-domain spectroscopy (THz-TDS) of pure, mixed, and layered ices of simple species $\left(\mathrm{CO}_{2}, \mathrm{H}_{2} \mathrm{O}\right),{ }^{16}$ as well as more complex species $(\mathrm{HCOOH}$, $\mathrm{CH}_{3} \mathrm{COOH}, \mathrm{CH}_{3} \mathrm{CHO}, \mathrm{CH}_{3} \mathrm{OH}$, and $\left.\left(\mathrm{CH}_{3}\right)_{2} \mathrm{CO}\right) .{ }^{17}$ Here, we present a comprehensive study of $\mathrm{CO}_{2}-\mathrm{CH}_{3} \mathrm{OH}$ mixtures in crystalline ices. We examine the role of segregation within the ices on the spectra at various mixing ratios, and discuss the possible impacts on the utility of these spectra for comparisons to observations.

\section{Experimental methods}

The underlying principles of the experiment, as well as the technical details of the instrument, have been described in detail elsewhere, ${ }^{16,17}$ and a schematic is shown in Fig. 1. Briefly, a35 fs, pulsed Ti:Sapphire regenerative amplifier at $800 \mathrm{~nm}$ drives an optical parametric amplifier (OPA) producing $1745 \mathrm{~nm}$ radiation in the idler beam. A portion of this radiation is co-linearly doubled in a beta-barium borate (BBO) crystal, and the two pulses are focused in a dry $\mathrm{N}_{2}$ purge, sparking a two-color plasma which produces intense, broadband THz radiation. ${ }^{23}$ The THz light is then focused through the sample, recombined with a portion of the original $800 \mathrm{~nm}$ pulse in a gallium phosphide $(\mathrm{GaP})$ crystal, and detected via free-space electro-optic sampling. ${ }^{24}$ In this arrangement, the spectrometer provides coverage from $\sim 0.3-7.0 \mathrm{THz}$. Data were collected for $30 \mathrm{ps}$, producing an experimental resolution of $\sim 0.03 \mathrm{THz}\left(1 \mathrm{~cm}^{-1}\right)$ when Fourier-transformed. A commerciallyavailable Fourier-transform infrared (FTIR) spectrometer provides simultaneous coverage from $\sim 500-4000 \mathrm{~cm}^{-1}$, also at $1 \mathrm{~cm}^{-1}$ resolution.

To prepare the ices, gas-phase samples of $\mathrm{CH}_{3} \mathrm{OH}$ and $\mathrm{CO}_{2}$ were first mixed in the desired ratios in a $1 \mathrm{~L}$ glass bulb attached to the dosing line. The pressures of each gas were monitored by a mass-independent pressure gauge. Gas-phase $\mathrm{CH}_{3} \mathrm{OH}$ was obtained by allowing a liquid sample of $299.9 \% \mathrm{CH}_{3} \mathrm{OH}$ (Sigma-Aldrich), which had been subjected to several freeze-pump-thaw cycles, to volatilize. High-purity $\mathrm{CO}_{2}$ from Air Liquide was used without further purification. Once prepared and mixed, the samples were introduced into the chamber via an all-metal leak valve, typically at a rate of $\sim 3.5 \mathrm{mTorr} \mathrm{s}^{-1}$, to the desired total pressure $\left(P_{\mathrm{tot}}\right)$, where they were frozen onto a high-resistivity $\mathrm{Si}$ substrate held at $T_{\mathrm{dep}}=80$ $\mathrm{K}$. In this high vacuum system, our ices are typically of order $10^{4}$ monolayers (ML) thick. After deposition, the samples were immediately cooled to a substrate temperature of $T_{\text {sub }}=$ $10 \mathrm{~K}$, and spectra collected at $10 \mathrm{~K}, 20 \mathrm{~K}$, and $30 \mathrm{~K}$, followed by annealing, typically for $\sim 5$ minutes, to $T_{\text {ann }}=90 \mathrm{~K}, 120 \mathrm{~K}$, and $140 \mathrm{~K}$. After each annealing, the samples were cooled to $10 \mathrm{~K}$ and spectra collected before the next annealing. A detailed list of experiments is given in Table 1.

\section{Results}

We have previously reported on the temperature-dependent spectra of pure end-member crystalline methanol (hereafter $\mathrm{c}-\mathrm{CH}_{3} \mathrm{OH}$ ) ${ }^{17}$ but have re-measured the spectra for this study 
under identical temperature and annealing conditions for consistency. Fig. 2 shows that while c- $\mathrm{CH}_{3} \mathrm{OH}$ ice is characterized by a series of sharper bands between $2-6 \mathrm{THz}$, amorphous methanol $\left(\mathrm{a}-\mathrm{CH}_{3} \mathrm{OH}\right)$ is largely characterized by a broad feature around $4.3 \mathrm{THz}$, and the beginning of a second, broad signal around $6 \mathrm{THz}$. Like the a-CH $\mathrm{CH}_{3} \mathrm{OH}$ shown in Fig. 2 , the a- $\mathrm{CH}_{3} \mathrm{OH}$ ice generated in this study via deposition at $80 \mathrm{~K}$ displays only a single, broad absorption across the $0.5-7 \mathrm{THz}$ window. An instrumental artifact around $\sim 2.1 \mathrm{THz}$ is also seen in some scans. The sharper, characteristic signals from $\mathrm{c}-\mathrm{CH}_{3} \mathrm{OH}$ become evident after annealing at $140 \mathrm{~K}$, when sufficient energy is available to enable crystallization. In a few cases, most prominently those where $\mathrm{CH}_{3} \mathrm{OH}$ strongly dominates the $\mathrm{CO}_{2}$, some c$\mathrm{CH}_{3} \mathrm{OH}$ features are seen at $120 \mathrm{~K}$. Notably, there does appear to be some weak signal from c- $\mathrm{CH}_{3} \mathrm{OH}$ after the $120 \mathrm{~K}$ annealing step in the most dilute $(10: 1) \mathrm{CO}_{2}: \mathrm{CH}_{3} \mathrm{OH}$ mixture.

Our first study of pure crystalline carbon dioxide $\left(\mathrm{c}-\mathrm{CO}_{2}\right)$ was reported in Allodi et al., ${ }^{16}$ but a lack of sensitivity and resolution in these initial experiments showed no clear features, despite a reported prior observation of a feature at $\sim 3.3 \mathrm{THz}$ in the literature. ${ }^{25}$ More recently, Giuliano et al. ${ }^{26}$ reported an observation of the $3.3 \mathrm{THz}$ feature, as well as an additional feature around $2.1 \mathrm{THz}$. However, they attribute these signals to amorphous carbon dioxide $\left(\mathrm{a}-\mathrm{CO}_{2}\right)$ rather than $\mathrm{c}-\mathrm{CO}_{2}$. We have recently conducted a thorough investigation of a- $\mathrm{CO}_{2}$ and $\mathrm{c}-\mathrm{CO}_{2}$ features in this frequency range. In brief, we find unambiguous evidence that these two features are due solely to c- $\mathrm{CO}_{2}$, and that a- $\mathrm{CO}_{2}$ shows no distinct features within the coverage of our spectrometer. For the purposes of this study, we have repeated the measurements of pure $\mathrm{CO}_{2}$ under the same experimental conditions used for the mixtures. For comparison, we also present a spectrum of a- $\mathrm{CO}_{2}$ from the forthcoming Ioppolo et al. publication (Fig. 2). We note that the sensitivity and resolution of the Caltech spectrometer has significantly improved in the two years since the publication of Allodi et al. ${ }^{16}$ through a combination of instrumental upgrades.

Spectra collected for this work are shown in Fig. 3. The reduction procedure has been described in detail previously. ${ }^{17}$ Briefly, a fast Fourier transform of the time-domain data is performed after applying an asymmetric Hann window to the data. This converts the spectra to the frequency domain. Baselines were then removed from the spectra by fitting the linefree regions to either a static offset or 1st-order linear fit, although the later was rarely required.

\section{$3.1 \quad \mathrm{CO}_{2}$-dominated mixtures}

Signals from the $2.1 \mathrm{THz}$ and $3.5 \mathrm{THz}$ c- $\mathrm{CO}_{2}$ modes are clearly seen in the 1:1, 3:1, and 10: 1 mixtures until annealing at $140 \mathrm{~K}$. In the case of pure $\mathrm{c}-\mathrm{CO}_{2}$, these features disappear after annealing at $120 \mathrm{~K}$, unlike in the mixed cases. Signal from c- $\mathrm{CH}_{3} \mathrm{OH}$ is clearly seen after annealing to $140 \mathrm{~K}$ in all three mixtures, at which point no $\mathrm{c}-\mathrm{CO}_{2}$ is apparent, although the near-coincidence of the $\mathrm{c}-\mathrm{CO}_{2}$ features with two $\mathrm{c}-\mathrm{CH}_{3} \mathrm{OH}$ features makes this determination somewhat ambiguous. For the 3:1 and 10:1 mixtures, features from c$\mathrm{CH}_{3} \mathrm{OH}$, particularly at $\sim 5.2 \mathrm{THz}$ and $\sim 2.6 \mathrm{THz}$, do begin to appear after annealing at 120 $\mathrm{K}$. The same features are possibly present in the $1: 1$ mixture, but would be just above the noise floor (baseline noise) if real. 
Fig. 4 (top) shows a quantitative analysis of the linewidths of the observed c- $\mathrm{CO}_{2}$ transitions, as determined by a Gaussian fit to the features. Due to the relatively low signal-to-noise ratio (SNR) of the lines, fits to the $10 \mathrm{~K}, 30 \mathrm{~K}$, and $60 \mathrm{~K}$ signals were averaged to determine the linewidth, with the standard deviation of this average given as the error bars. While some broadening of the transitions was observed with temperature, this broadening was not linear over the entire range of mixing ratios. Instead, the linewidth appears to depend heavily on mixing ratio. The results show a significant increase in line- width between pure $\mathrm{CO}_{2}$ and mixed ices (Fig. 4). For the pure ice, the $2.1 \mathrm{THz}$ transition is substantially narrower than the $3.5 \mathrm{THz}$ transition. The mixed ices, conversely, have a largely uniform linewidth regardless of mixing ratio (within the uncertainties). They are also all significantly broader than either transition in pure $\mathrm{CO}_{2}$. The increase in linewidth between the pure and mixed $2.1 \mathrm{THz}$ transitions, however, is markedly greater on average compared to the $3.5 \mathrm{THz}$ transition: factors of 4.5 vs. 2.0, respectively.

Fig. 4 (bottom) shows a quantitative analysis of the linewidths of the observed $\mathrm{CO}_{2}$ transitions in the FTIR shown in Fig. 5 (middle), as determined by a Guassian fit to the features. Because of the much higher SNR, only the $10 \mathrm{~K}$ scan with no annealing was used for the determination, and the errors are purely due to uncertainty in the Gaussian fit. As in the THz, the mid-IR linewidths show a mixing ratio dependence. The magnitude of the change is about half that in the $\mathrm{THz}$, and unlike the $\mathrm{THz}$, seems to increase linearly for both transitions from pure $\mathrm{CO}_{2}$ to the $3: 1$ mixture. Finally, while the linewidths for the $1: 1$ mixture in the THz remain similar to those of the 10: 1 and $3: 1$ mixtures, in the FTIR the 1 : 1 and $1: 3$ mixtures are significantly narrower.

\section{$3.2 \mathrm{CH}_{3} \mathrm{OH}$-dominated mixtures}

The $\mathrm{THz}$ signatures of $\mathrm{c}-\mathrm{CO}_{2}$ appear to be strongly suppressed in both $\mathrm{CH}_{3} \mathrm{OH}$-dominated mixtures. A very weak indication of the $2.1 \mathrm{THz}$ c- $-\mathrm{CO}_{2}$ may be visible in a handful of scans, but it is difficult to distinguish from both the weak artifact (introduced by the HDPE beam block) in this region, and the overlapping $\mathrm{c}-\mathrm{CH}_{3} \mathrm{OH}$ mode which begins to appear after crystallization starts at $120 \mathrm{~K}$. Of the mixtures studied, only pure $\mathrm{CH}_{3} \mathrm{OH}$ and the $3: 1 \mathrm{CO}_{2}$ dominated mixture show a clear separation of the two $\mathrm{c}-\mathrm{CH}_{3} \mathrm{OH}$ transitions around $2.6 \mathrm{THz}$. The others show only a single blended peak in this region.

\section{Discussion}

After $\mathrm{H}_{2} \mathrm{O}$ and $\mathrm{CO}, \mathrm{CO}_{2}$ is one of the most prominent ice species in the ISM, with abundances of nearly $20 \%$ that of $\mathrm{H}_{2} \mathrm{O}$ ice. ${ }^{27,28}$ In dense molecular clouds, $\mathrm{CO}_{2}$ is formed in the solid phase primarily through the $\mathrm{CO}+\mathrm{OH}$ reaction that has been experimentally found to be 10 times more efficient than the $\mathrm{CO}+\mathrm{O}$ channel. ${ }^{29}$ Therefore, although $\mathrm{CO}$ is its parent molecule, $\mathrm{CO}_{2}$ tends to reside primarily in polar ices (i.e. $\mathrm{H}_{2} \mathrm{O}$-rich rather than $\mathrm{CO}$ rich), where $\mathrm{OH}$ radicals are more abundant and available for reaction with $\mathrm{CO}$. Thus, midIR and $\mathrm{THz}$ spectroscopic studies of $\mathrm{CO}_{2}$ in polar environments are important to our understanding of the origin and evolution of interstellar $\mathrm{CO}_{2}$ ices. ${ }^{16,30}$

Outside of mixtures with $\mathrm{H}_{2} \mathrm{O}, \mathrm{CH}_{3} \mathrm{OH}$ is the most abundant polar ice constituent at $\sim 6-9 \%$ of the abundance of $\mathrm{H}_{2} \mathrm{O}$, or $\sim 20-50 \%$ of the abundance of $\mathrm{CO}_{2} \cdot{ }^{13} \mathrm{CO}$ and $\mathrm{CO}_{2}$ have been 
shown to be the products of $\mathrm{UV}$ and cosmic ray irradiation of $\mathrm{CH}_{3} \mathrm{OH}$-containing ices. ${ }^{31}$ Therefore, in later stages of star formation, when ices are extensively exposed to heating, UV photons, and cosmic rays, $\mathrm{CO}_{2}$ is thermally processed and mixed with $\mathrm{CH}_{3} \mathrm{OH} .{ }^{32}$ As the segregation of $\mathrm{CO}_{2}$ into ordered crystalline micro-domains is thought to be a powerful probe of such thermal processing in astrophysical environments, ${ }^{30,33}$ it is interesting to explore that segregation in the laboratory, and its observational implications.

\subsection{Structure and segregation}

The segregation of $\mathrm{CO}_{2}$ ice upon deposition at warm temperatures has been previously reported in mixed $\mathrm{CO}_{2}-\mathrm{H}_{2} \mathrm{O}^{34}$ and $\mathrm{CO}_{2}-\mathrm{H}_{2} \mathrm{O}-\mathrm{CH}_{3} \mathrm{OH}^{28}$ ices in studies with mid-IR spectroscopy. One of the many advantages of THz-TDS in studying ices, however, is that the features arising from these species in the $\mathrm{THz}$ regime result from the collective motion of many molecules (inter-molecular modes) and thus serve as a direct probe of the structure of the ice. ${ }^{16,17}$ This is in contrast to mid-IR spectroscopy, where the ice structure must be indirectly inferred from changes in the lineshape of intramolecular modes.

This utility is immediately obvious from the observations of the linewidths of the $\mathrm{c}-\mathrm{CO}_{2}$ features within the various mixtures. While the features from pure $\mathrm{c}-\mathrm{CO}_{2}$ are relatively sharp, any amount of contamination from $\mathrm{CH}_{3} \mathrm{OH}$ significantly broadens the transitions. This demonstrates how the $\mathrm{THz}$ transitions of $\mathrm{CO}_{2}$ are useful as a probe of local structure. As these are solid-phase materials that do not have structural rearrangement happening of a timescale faster than our measurement, the broadening of the observed spectra features results from the different ice environments experienced by the $\mathrm{CO}_{2}$ molecules, and can be correctly characterized as inhomogenously broadened. Even a $10 \% \mathrm{CH}_{3} \mathrm{OH}$ contamination is apparently sufficient to create a variety of local environments within the ice, and inhomogenously broaden these transitions.

Since the spectral features at $\mathrm{THz}$ frequencies are inter-molecular in nature, the amount of inhomogenous broadening offers a direct measure of the number of unique structural environments present in the ice. While the inhomogenous broadening of intra-molecular modes provides insight into the number of different local environments of individual molecules, the inhomogenous broadening of inter-molecular modes can only happen when there are many different structural environments, leading to differences in the frequency of the collective motion of many molecules.

Interestingly, there is a lack of a clearly increasing trend in linewidth in the $\mathrm{THz}$ observations, unlike those in the FTIR. If we assume that the linewidth from pure c- $\mathrm{CO}_{2}$ represents the homogenous value, this suggests that the $\mathrm{CO}_{2}-\mathrm{CH}_{3} \mathrm{OH}$ interaction is somewhat uniform across mixing ratios. It is possible that the data show a trend of increasing linewidth with increasing $\mathrm{CO}_{2}$ concentration within the ice, but this cannot be claimed definitively given the uncertainties in the measurements. Follow-up studies will examine this effect, as well as providing an in-depth examination of the degree of segregation and the size of the $\mathrm{c}-\mathrm{CO}_{2}$ domains under various conditions. Finally, the larger degree of broadening observed in the $2.1 \mathrm{THz}$ transition, relatively to the $3.5 \mathrm{THz}$ transition, may provide insight into the nature of these motions in the bulk ice. 
Hodyss et al. ${ }^{34}$ observed that features of $\mathrm{c}-\mathrm{CO}_{2}$ in their $\mathrm{CO}_{2}-\mathrm{H}_{2} \mathrm{O}$ mixtures initially appear at $60 \mathrm{~K}$, are distinct by $70 \mathrm{~K}$, and gradually lessen as the ice is heated further to $80 \mathrm{~K}$ and disappear at $100 \mathrm{~K}$ as the $\mathrm{CO}_{2}$ sublimates. In initially-amorphous $\mathrm{CO}_{2}-\mathrm{CH}_{3} \mathrm{OH}$ ices studied by Ehrenfreund et al., ${ }^{28} \mathrm{CO}_{2}$ persists in the mixture at temperatures as high as $125 \mathrm{~K}$, and additional spectroscopic features emerge. These features are likely interactions between $\mathrm{CO}_{2}$ which has crystallized to a degree at lower temperatures, and the $\mathrm{CH}_{3} \mathrm{OH}$, which does not begin to crystallize until $\sim 120 \mathrm{~K}$. This is supported in our data, especially in the $3: 1$ mixture after annealing to $120 \mathrm{~K}$, where strong $\mathrm{c}-\mathrm{CO}_{2}$ features remain visible while c$\mathrm{CH}_{3} \mathrm{OH}$ signal begins to emerge. Indeed, although the $\mathrm{THz}$ signals of $\mathrm{c}-\mathrm{CO}_{2}$ are obscured by those of $\mathrm{c}-\mathrm{CH}_{3} \mathrm{OH}, \mathrm{c}-\mathrm{CO}_{2}$ is still present in the ice even after annealing to $140 \mathrm{~K}$, as indicated by FTIR spectra recorded contemporaneously (Fig. 6).

Interestingly, three sharp features are observed in the THz spectra of the $3: 1$ mixture around $2.2-2.8 \mathrm{THz}$, after the mixture has been annealed to $140 \mathrm{~K}$. While these features might be present as broad shoulders in the pure $\mathrm{CH}_{3} \mathrm{OH}$ ice after the same heating process, they are not nearly as distinct. Perhaps, as was the case with the $\mathrm{CO}_{2}$ stretch at $2340 \mathrm{~cm}^{-1}$ observed by Ehrenfreund et al., ${ }^{28}$ this is an indication of a coupling between the $\mathrm{CO}_{2}$ and $\mathrm{CH}_{3} \mathrm{OH}$ motions.

In the $\mathrm{CH}_{3} \mathrm{OH}$-dominated mixtures, no clear signal from c- $\mathrm{CO}_{2}$ is observed in any of the THz spectra, and while the bright $v_{3}$ band is always visible (and saturated) in the FTIR, the combination modes are heavily suppressed in the $\mathrm{CH}_{3} \mathrm{OH}$-dominated mixtures (Fig. 5 middle and bottom). While the FTIR spectra can distinguish between crystalline and amorphous $\mathrm{CO}_{2}$ under most circumstances (Fig. 5 top), the THz spectra offer a more powerful probe of segregation within the ice. The presence of $\mathrm{c}-\mathrm{CO}_{2}$ features would unambiguously indicate the presence of significantly-sized micro-domains of ordered $\mathrm{CO}_{2}$. The lack of any such features indicates that little to no aggregation of $\mathrm{CO}_{2}$ domains from within the $\mathrm{CH}_{3} \mathrm{OH}$, beyond the initial segregation at deposition, has occurred. This agrees with the conclusion of Ehrenfreund et al. ${ }^{28}$ that the $\mathrm{CH}_{3} \mathrm{OH}$-dominated ices are more thermally-stable, and less subject to reorganization with heating.

Finally, the degree of inhomogeneous broadening of the $\mathrm{c}-\mathrm{CO}_{2}$, relative to a pure, bulk ice, is an indicator of the variety of size scales of the crystalline micro-domains within the bulk ice. As the segregated $\mathrm{c}-\mathrm{CO}_{2}$ approaches a uniform size, and nears the formation of a single bulk crystal, this broadening should reduce to the narrower profiles of the pure c- $\mathrm{CO}_{2}$. A follow-up study could potentially monitor the change in broadening as a function of time while the ice is gently heated and $\mathrm{CO}_{2}$ segregation occurs, offering a direct probe of migration and crystallization timescales and dynamics within the ice. Further, as the broadening for the $3.5 \mathrm{THz}$ transition relative to the $2.1 \mathrm{THz}$ transition does not appear to be unity, this ratio could be used in astronomical observations to probe the level of segregation, even without a baseline, purely c- $\mathrm{CO}_{2}$ signature within the source to set the intrinsic width.

\subsection{Mixture-dependent frequencies}

As discussed above, the change in lineshape with mixing ratio in the $\mathrm{THz}$ spectra is the most obvious indicator of the effect of the bulk environment on the spectra. In the FTIR spectra, however, this effect is more readily apparent in the observed frequencies of the transitions. 
This is most clear in the case of $\mathrm{CO}_{2}$. For example, the $v_{3}$ band of $\mathrm{CO}_{2}$ shows a distinct blue-shift as the mixing ratio tends toward pure $\mathrm{CO}_{2}$ (Fig. 5 bottom). While the ${ }^{12} \mathrm{CO}_{2}$ feature is too saturated due to the thickness required for this experiment for a quantitative analysis, the ${ }^{13} \mathrm{CO}_{2}$ peak presents a large, $8.7 \mathrm{~cm}^{-1}$ shift from the $1: 10$ to pure $\mathrm{c}^{-\mathrm{CO}_{2}}$ ices. An analogous shift has been previously observed for clusters of $\mathrm{CO}_{2}$ in the gas phase. Here, the increasingly large clusters are essentially a shift toward the purely crystalline $\mathrm{CO}_{2}$ domain, and even larger blue shifts $\left(\sim 21 \mathrm{~cm}^{-1}\right)$ are observed as the cluster grows from the monomer to $N=13 .{ }^{37}$

We also note the possibility that signals could arise in the FTIR spectra from combination bands of the long-range, inter-molecular modes in the $\mathrm{THz}$ region and the intra-molecular motions in the infrared. These modes would in theory be distinguishable from simple isotopically-shifted intra-molecular modes by their frequency shifts: such isotopic shifts would be to the red, while combination bands would lie primarily to the blue of the monomer features. Because our ices are by necessity so thick, and the resulting monomer features saturated, it is likely that the lowest-lying of the combination bands will be buried beneath the overly-wide monomer signal. Nevertheless, the relatively sharp transitions observed in the THz should have counterparts in the IR, although the frequencies will be shifted and the lineshapes altered due to the effects of vibrational excitation and different coupling to the bulk environment. Isotopic labeling, which would differentially affect the intra- $v s$. inter-molecular modes and thus make combination bands distinct from monomer features, is a promising avenue to exploring this potential interaction. Such studies are beyond the scope of the current work, however.

\subsection{Observational implications}

In terms of detectability, it seems likely that features of $\mathrm{c}-\mathrm{CO}_{2}$ will be present in astronomical observations, assuming it is sufficiently segregated both from smaller contaminant species, such as the $\mathrm{CH}_{3} \mathrm{OH}$ studied here, and from the polar $\mathrm{H}_{2} \mathrm{O}$ ices where it is formed. This segregation has already been observed astronomically. ${ }^{30,33,38}$ It follows that the $\mathrm{THz}$ signals of $\mathrm{c}-\mathrm{CO}_{2}$ would be excellent targets for interstellar observations, as they are unambiguous evidence of segregated, $\mathrm{c}-\mathrm{CO}_{2}$. Given the extensive broadening of the $\mathrm{THz}$ modes when the $\mathrm{c}-\mathrm{CO}_{2}$ is in a mixture, it is also possible that such a width could be used as an indicator of the degree of segregation within these inter-stellar ices. Further work will certainly be needed to determine whether this is truly a useful probe, especially at mixing ratios higher than those used in this initial study (i.e. > $10: 1$ ).

Interestingly, the $\mathrm{THz}$ features of $\mathrm{CH}_{3} \mathrm{OH}$ are also distinct after annealing at $120 \mathrm{~K}$, even in both mixtures (10:1 and $3: 1)$ that clearly still contain substantial fractions of $\mathrm{CO}_{2}$ (see Fig. 7). Indeed, the $\mathrm{c}-\mathrm{CH}_{3} \mathrm{OH}$ features are far stronger than the $\mathrm{c}-\mathrm{CO}_{2}$, although broader. Their presence in an interstellar observation would therefore indicate the thermal history of the ices, while the peak positions of the features have already been shown to be dependent on the observed temperature of the ice. ${ }^{16,17}$

An important consideration is the role of dust grain size and composition underlying interstellar ices, and the impact these have on the ice structure and subsequently their spectra. Indeed, recent modeling work by Pauly and Garrod ${ }^{39}$ has shown that while the 
chemical makeup of the ices does not greatly vary with grain size, there can be significant stratification in which size-class of grains is the dominant ice carrier. For example, during cloud collapse, they find that small grains dominate as ice carriers, resulting in ice thickness of $<40 \mathrm{ML}$, versus those of order $\sim 10^{2}$ if the model includes only a single grain size. This raises the important question: to what degree can species segregate within these ices, and how will that affect the spectra they present?

Due to the sensitivity of the second-generation THz spectrometer presented here, we could only study thick laboratory ices (of order $\sim 10^{4} \mathrm{ML}$ ). The current-generation spectrometer is now capable of studying ices of order $\sim 10^{3} \mathrm{ML}$, and the next-generation instrument should push into the $10^{1}-10^{2} \mathrm{ML}$ regime with the ability to directly measure the optical constants of the ices under investigation. This is because the electro-optic sampling technique employed here directly measures both the amplitude and phase of the $\mathrm{THz}$ pulse. This presents a experimental approach which is far simpler than complementary techniques with an FTIR that require an asymmetric configuration where the sample resides in an arm of the interferometer. ${ }^{40}$ Combined with molecular dynamics simulations, radiative transfer, and scattering models over a realistic range in dust particle size, these optical constant measurements will allow for far more accurate modeling of observational spectra. Such models will in turn provide even more precise information on not just the ice composition and structure, but that of the underlying grain substrate. With the critical role that the size of icy grains has on the growth of macroscopic grains and gaps in protoplanetary disks, ${ }^{41}$ this information will be essential for understanding physical evolution in star and planet formation.

Indeed, factors such as composition, physical structure and segregation, temperature, and thermal history all play crucial roles in the evolution of molecular complexity both in molecular clouds and in evolving planetary systems. ${ }^{5}$ Observations of ices in the $\mathrm{THz}$ regime offer the potential to shed light on these factors, and in the case of crystallinity and segregation, to do so unambiguously due to the nature of the THz modes. Molecular ices are the birthplace of the prebiotic complexity which will eventually be incorporated into nascent solar systems, and thus understanding the environments in which they form, and the mechanisms of formation, is critical to understanding the genesis of this primordial material.

\section{Acknowledgements}

The authors thank P. Carroll, I. Finneran, and M. Kelley for their assistance with spectrometer development. B. A. M. thanks A. Remijan for his support. The authors thank the anonymous referee for a detailed reading of the manuscript which significantly improved the quality of the work. S. I. acknowledges funding through a Marie Curie Fellowship (FP1-PEOPLE-2011-IOF-300957) and recent support by the Royal Society. M. A. A. acknowledges current support from a Yen Postdoctoral Fellowship from the Institute for Biophysical Dynamics at the University of Chicago. The National Radio Astronomy Observatory is a facility of the National Science Foundation operated under cooperative agreement by Associated Universities, Inc.

\section{References}

1. Chyba CF, Thomas PJ, Brookshaw L. and Sagan C, Science, 1990, 249, 366-373. [PubMed: 11538074]

2. Cleeves LI, Bergin EA, Alexander CMO, Du F, Graninger D, Öberg KI and Harries TJ, Science, 2014, 345, 1590-1593. [PubMed: 25258075] 
3. Belloche A, Müller HSP, Menten KM, Schilke P. and Comito C, Astron. Astrophys, 2013, 559, A47-187.

4. Herbst E. and van Dishoeck EF, Annu. Rev. Astron. Astrophys, 2009, 47, 427-480.

5. Garrod RT, Astrophys. J, 2013, 765, 60.

6. Tielens A. and Hagen W, Astron. Astrophys, 1982, 245-260.

7. Charnley SB, Rodgers SD and Ehrenfreund P, Astron. Astrophys, 2001, 378, 1024-1036.

8. Watanabe N. and Kouchi A, Astrophys. J, 2002, 571, L173-L176.

9. Ioppolo S, Cuppen HM, Romanzin C, van Dishoeck EF and Linnartz H, Astrophys. J, 2008, 686, 1474.

10. Congiu E, Fedoseev G, Ioppolo S, Dulieu F, Chaabouni H, Baouche S, Lemaire JL, Laffon C, Parent P, Lamberts T, Cuppen HM and Linnartz H, Astrophys. J, 2012, 750, L12.

11. Laas JC, Garrod RT, Herbst E. and Weaver Widicus S. L., Astrophys. J, 2011, 728, 71.

12. Chuang KJ, Fedoseev G, Ioppolo S, van Dishoeck EF and Linnartz H, Mon. Not. R. Astron. Soc, 2016, 455, 1702-1712.

13. Boogert ACA, Gerakines PA and Whittet DCB, Annu. Rev. Astron. Astrophys, 2015, 53, 541-581.

14. Linnartz H, Ioppolo S. and Fedoseev G, Int. Rev. Phys. Chem, 2015, 34, 205-237.

15. Profeta G. and Scandolo S, Phys. Rev. B: Condens. Matter Mater. Phys, 2011, 84, 024103.

16. Allodi MA, Ioppolo S, Kelley MJ, McGuire BA and Blake GA, Phys. Chem. Chem. Phys, 2014, 16, 3442. [PubMed: 24394213]

17. Ioppolo S, McGuire BA, Allodi MA and Blake GA, Faraday Discuss, 2014, 168, 461-484. [PubMed: 25302394]

18. Palumbo ME, J. Phys. Chem. A, 1997, 101, 4298-4301.

19. Mastrapa RM, Sandford SA, Roush TL, Cruikshank DP and Ore Dalle C. M., Astrophys. J, 2009, 701, 1347-1356.

20. Jenniskens P. and Blake DF, Astrophys. J, 1996, 473, 1104-1113. [PubMed: 11539415]

21. Moore MH and Hudson RL, Astrophys. J, 1992, 401, 353-360.

22. McClure MK, Espaillat C, Calvet N, Bergin E, D' Alessio P, Watson DM, Manoj P, Sargent B. and Cleeves LI, Astrophys. J, 2015, 799, 162.

23. Clerici M, Peccianti M, Schmidt BE, Caspani L, Shalaby M, Giguère M, Lotti A, Couairon A, Lègaré F, Ozaki T, Faccio D. and Morandotti R, Phys. Rev. Lett, 2013, 110, 253901.

24. Wu Q. and Zhang XC, Appl. Phys. Lett, 1995, 67, 3523.

25. Moore MH and Hudson RL, Astron. Astrophys, 1994,103, 45-56.

26. Giuliano BM, Escribano RM, Martn-Doménech R, Dartois E. and Muñoz Caro GM, Astron. Astrophys, 2014, 565, A108.

27. Whittet D, Gerakines PA and Tielens A, Astrophys. J, 1998, 75, L159-L163.

28. Ehrenfreund P, Kerkhof O, Schutte WA, Boogert A, Gerakines PA, Dartois E, d'Hendecourt L, Tielens A, van Dishoeck PF and Whittet D, Astron. Astrophys, 1999, 350, 240-253.

29. Ioppolo S, Fedoseev G, Lamberts T, Romanzin C. and Linnartz H, Rev. Sci. Instrum, 2013, 84, 073112.

30. Pontoppidan KM, Boogert ACA, Fraser HJ, van Dishoeck EF, Blake GA, Lahuis F, Öberg KI, Evans II NJ and Salyk C, Astrophys. J, 2008, 678, 1005-1031.

31. Islam F, Baratta GA and Palumbo ME, Astron. Astrophys, 2014, 561, A73-A79.

32. Ioppolo S, Sangiorgio I, Baratta GA and Palumbo ME, Astron. Astrophys, 2013, 554, A34.

33. Kim HJ, Evans II NJ, Dunham MM, Lee J-E and Pontoppidan KM, Astrophys. J, 2012, 758, 38.

34. Hodyss R, Johnson PV, Orzechowska GE, Goguen JD and Kanik I, Icarus, 2008, 194, 836-842.

35. Lehmann B, Wahlen M, Zumbrunn R. and Oeschger H, Appl. Phys, 1977, 13, 153-158.

36. Dartois E. and Schmitt B, Astron. Astrophys, 2009, 504, 869-873.

37. Norooz Oliaee J, Dehghany M, McKellar ARW and Moazzen-Ahmadi N, J. Chem. Phys, 2011, $135,044315$.

38. Escribano RM, Muñoz Caro GM, Cruz-Diaz GA, Rodriguez-Lazcano Y. and Mate B, Proc. Natl. Acad. Sci. U. S. A, 2013, 110, 12899-12904.

Phys Chem Chem Phys. Author manuscript; available in PMC 2019 November 09. 
39. Pauly T. and Garrod RT, Astrophys. J, 2016, 817, 1-16.

40. Birch JR, Microchim. Acta, 1987, 93, 105-122.

41. Zhang K, Blake GA and Bergin EA, Astrophys. J., Lett, 2015, 806, 1-6. 


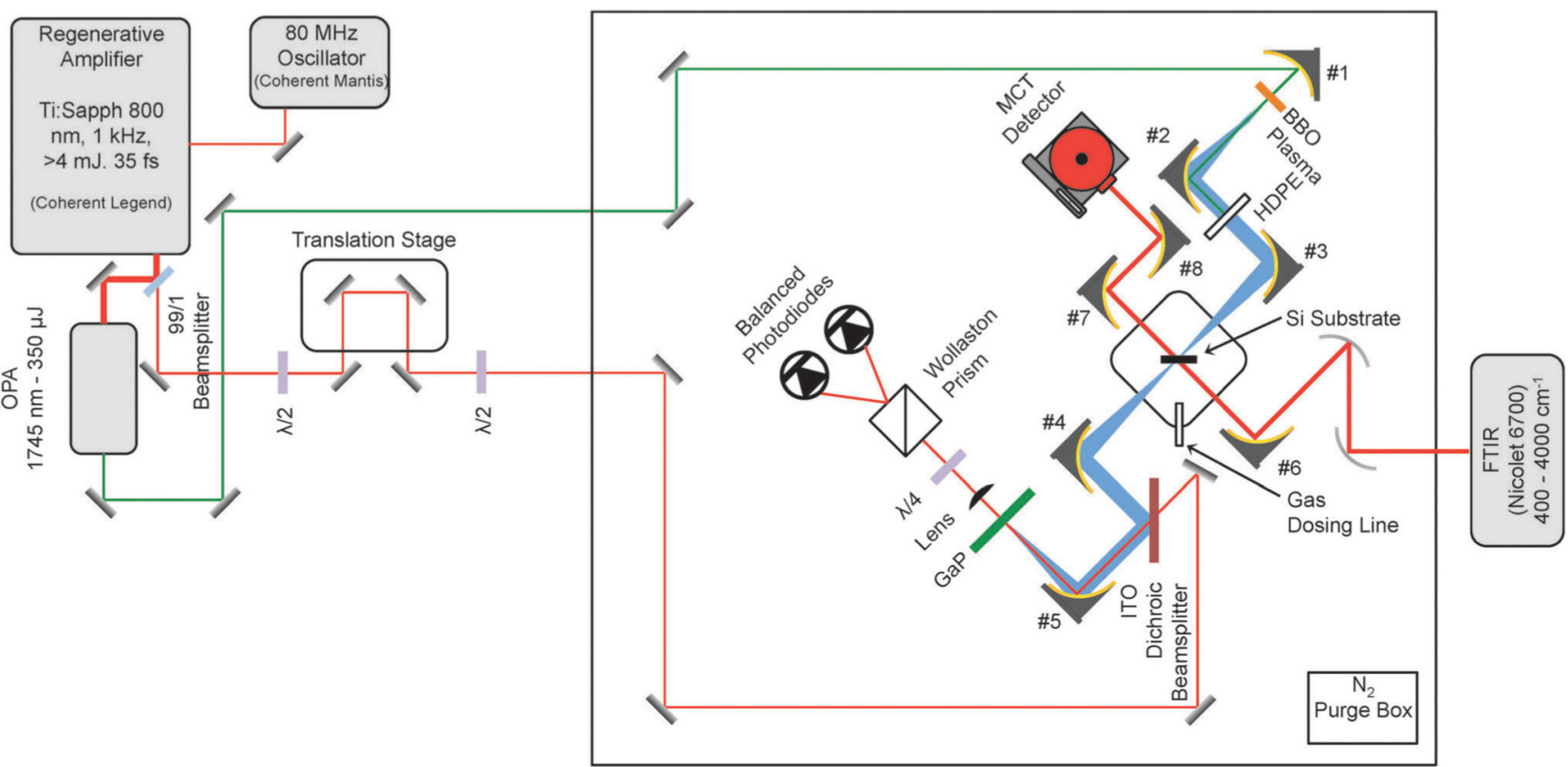

Fig. 1.

Schematic overview of the Caltech THz-TD spectrometer, and its application to the study of astrochemical ice analogs. The $800 \mathrm{~nm}$ output of the Legend oscillator is split, with a portion of the light passing through an optical parametric amplifier (OPA). This light is then doubled in a beta-barium borate (BBO) crystal and focused with an off-axis parabolic mirror (numbered optics) to spark a plasma. A high-density polyethylene (HDPE) beam block filters out the visible light, and after passing through the sample, the $\mathrm{THz}$ beam is recombined with the other $800 \mathrm{~nm}$ beam in an indium tin oxide (ITO) dichroic beamsplitter and focused onto a gallium phosphide $(\mathrm{GaP})$ crystal for detection. The infrared spectrometer signal is detected by a mercury cadmium telluride (MCT) detector. 

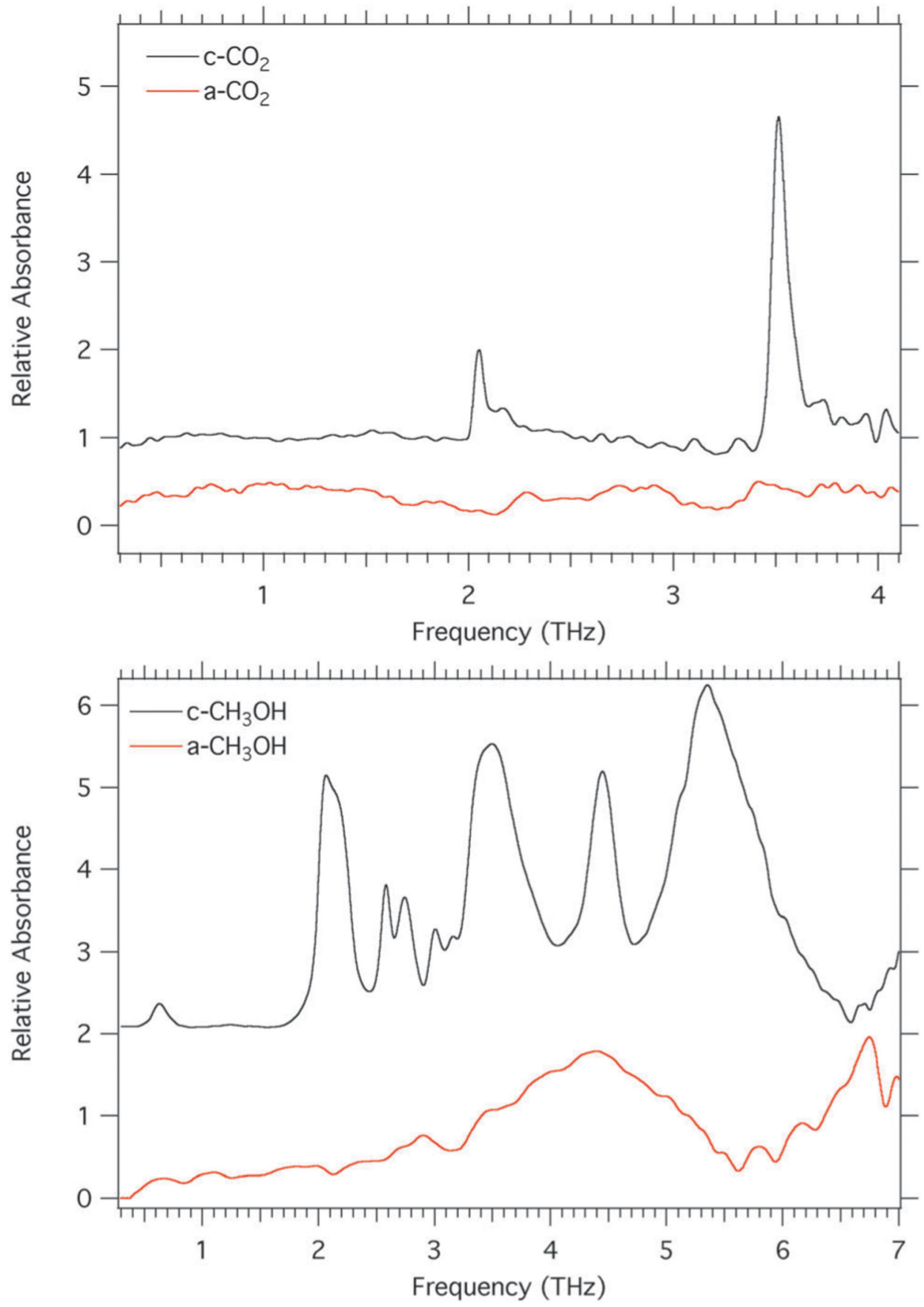

Fig. 2.

(top) Spectra of amorphous $\mathrm{CO}_{2}\left(\mathrm{a}-\mathrm{CO}_{2}\right)$ deposited at $10 \mathrm{~K}$ (red) and crystalline $\mathrm{CO}_{2}$ (c$\mathrm{CO}_{2}$ ) deposited at $80 \mathrm{~K}$ (black). Both spectra were acquired at $10 \mathrm{~K}$, and have been vertically-offset for clarity. (bottom) Spectra of a- $\mathrm{CH}_{3} \mathrm{OH}$ deposited at $10 \mathrm{~K}$ (red) and c$\mathrm{CH}_{3} \mathrm{OH}$ deposited at $140 \mathrm{~K}$ (black). Both spectra were taken at $10 \mathrm{~K}$, and vertically-offset for clarity. 

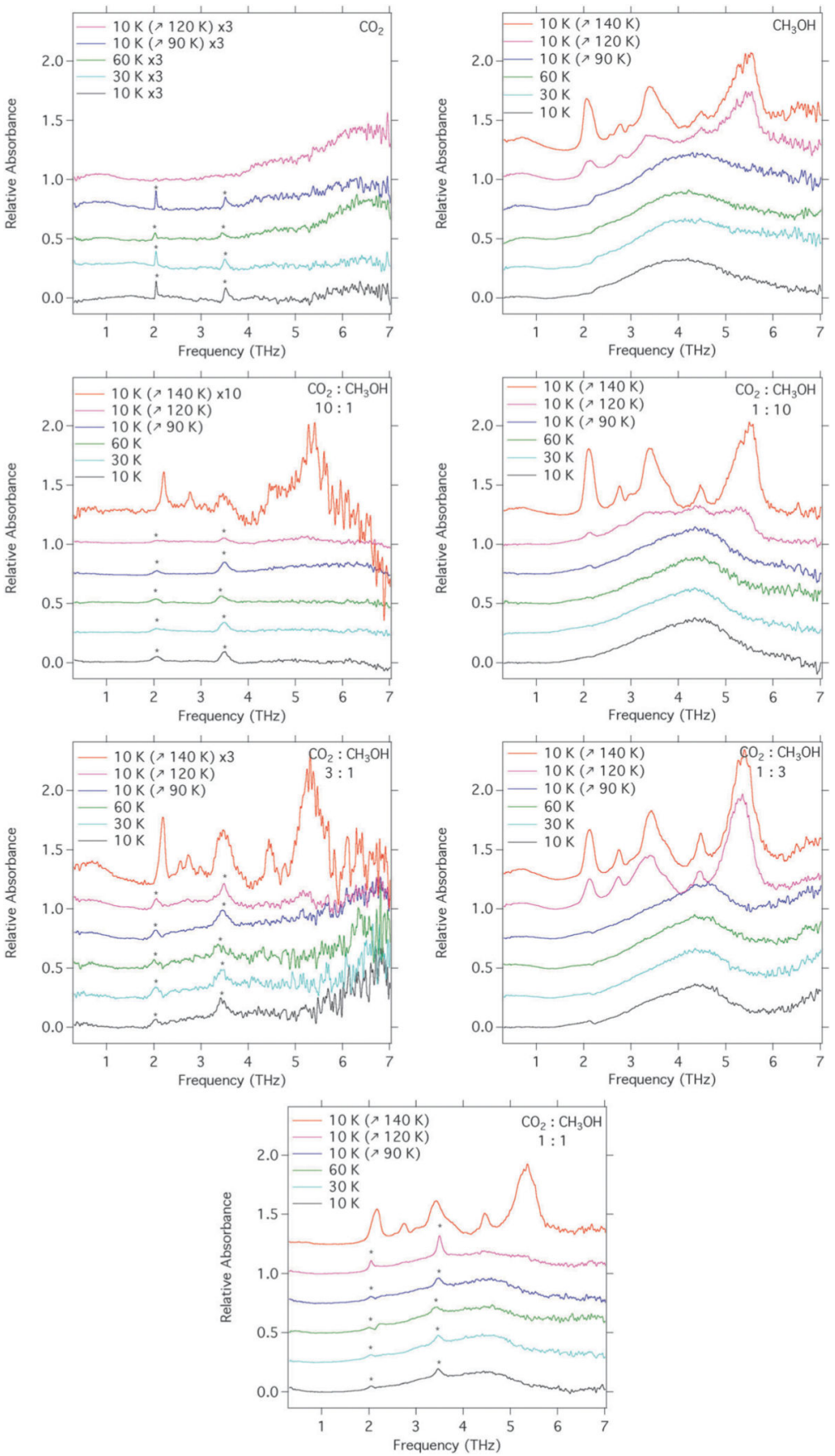

Fig. 3.

THz-TDS spectra collected for this study. All ices were deposited at $80 \mathrm{~K}$, and spectra were collected at the temperatures indicated in the caption $(10 \mathrm{~K}, 30 \mathrm{~K}$, and $60 \mathrm{~K})$. In cases where the ices were annealed, the annealing temperature is indicated by $\nearrow$. Ice compositions are given in the upper right of each panel. Spectra are vertically-offset for clarity, and, when noted, are scaled to show detail. The positions of the $\mathrm{c}-\mathrm{CO}_{2}$ features are marked by asterisks $(*)$ when present. 

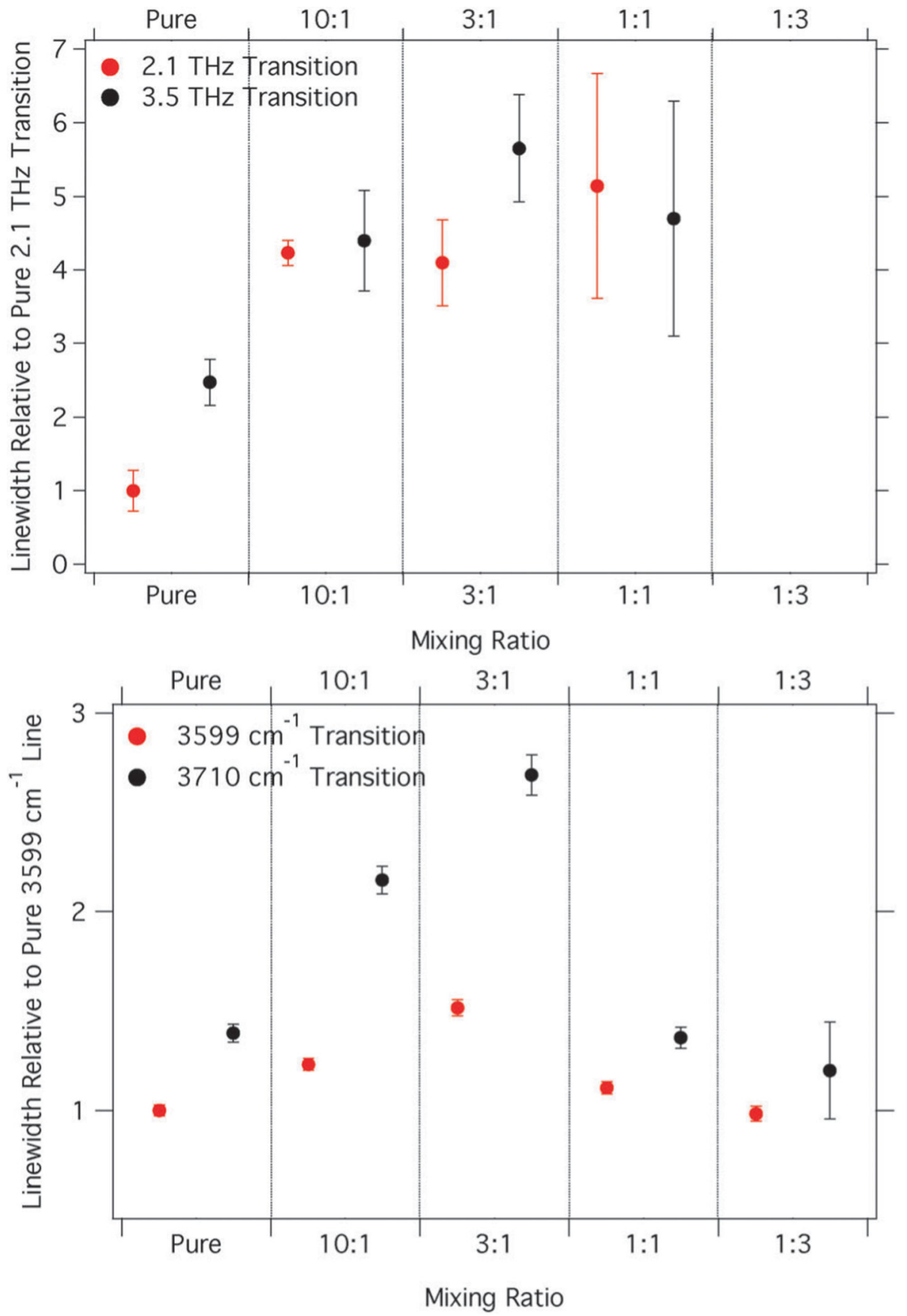

Fig. 4.

(top) Average FWHM values of the $2.1 \mathrm{THz}$ and $3.5 \mathrm{THz} \mathrm{c}-\mathrm{CO}_{2}$ transitions observed in this work at $10 \mathrm{~K}, 30 \mathrm{~K}$, and $60 \mathrm{~K}$ for pure $\mathrm{CO}_{2}$ and the indicated mixing ratios. Values are normalized to the pure, $2.1 \mathrm{THz}$ width. Error bars are $1 \sigma$ standard deviations in the averages. (bottom) FWHM values of the $3599 \mathrm{~cm}^{-1}$ and $3710 \mathrm{~cm}^{-1} 2 v_{2}+v_{3}$ and $v_{1}+v_{3}$, respectively, $\mathrm{CO}_{2}$ transitions at $10 \mathrm{~K}$ for pure $\mathrm{CO}_{2}$ and the indicated mixing ratios. 

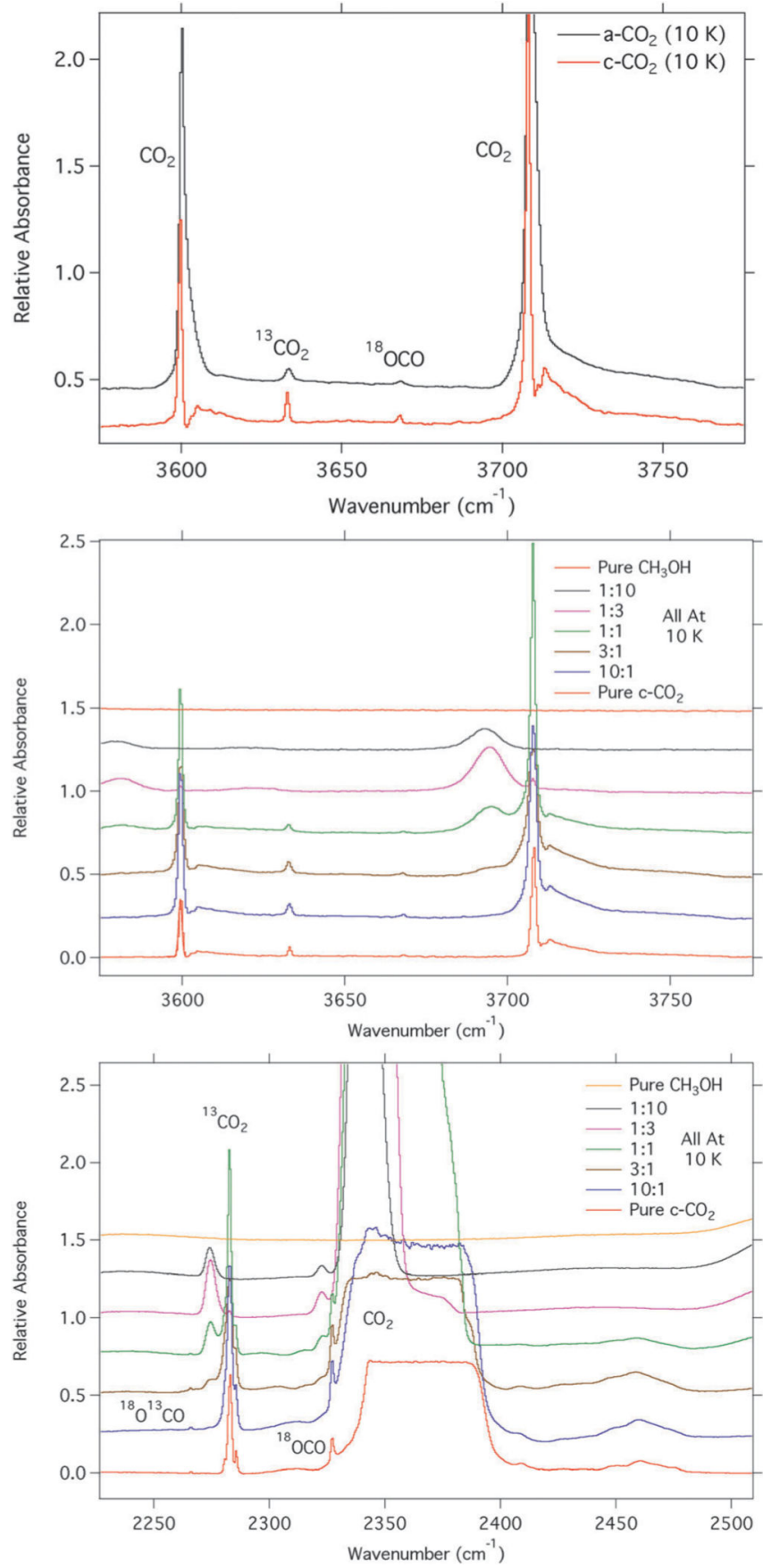

Fig. 5.

(top) FTIR spectra of the $v_{1}+v_{3}$ and $2 v_{2}+v_{3}$ combination bands of a- $\mathrm{CO}_{2}$ (black) and c$\mathrm{CO}_{2}$ (red) collected in our laboratory at $10 \mathrm{~K}$. (middle) The same modes collected during the $10 \mathrm{~K}$ experiment (no annealing) for each of the mixtures studied in this work. (bottom) The $v_{3}$ band of $\mathrm{CO}_{2}$ and ${ }^{13} \mathrm{CO}_{2}$ collected during the $10 \mathrm{~K}$ experiment (no annealing) for each of the mixtures studied in this work. Spectra are vertically-offset for clarity, and several additional features due to isotopologues are indicated. ${ }^{35,36}$ 


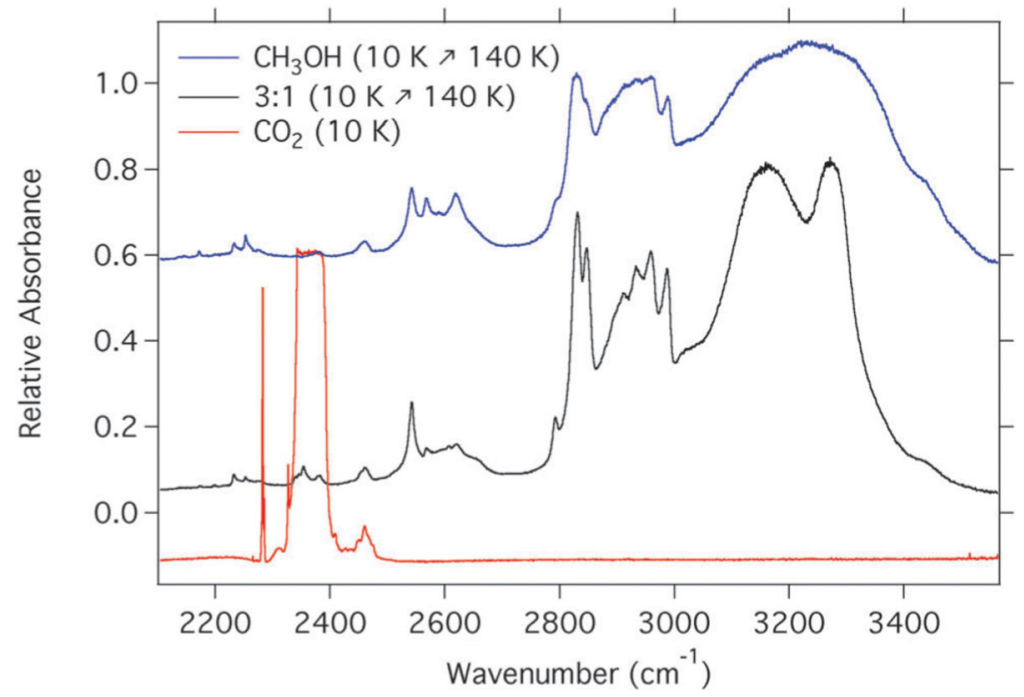

Fig. 6.

FTIR spectra of pure c- $\mathrm{CO}_{2}$ at $10 \mathrm{~K}$ (bottom), the $3: 1$ mixture at $10 \mathrm{~K}$ after annealing to 140 $\mathrm{K}$ (middle), and pure $\mathrm{CH}_{3} \mathrm{OH}$ at $10 \mathrm{~K}$ after annealing to $140 \mathrm{~K}$ (top). 


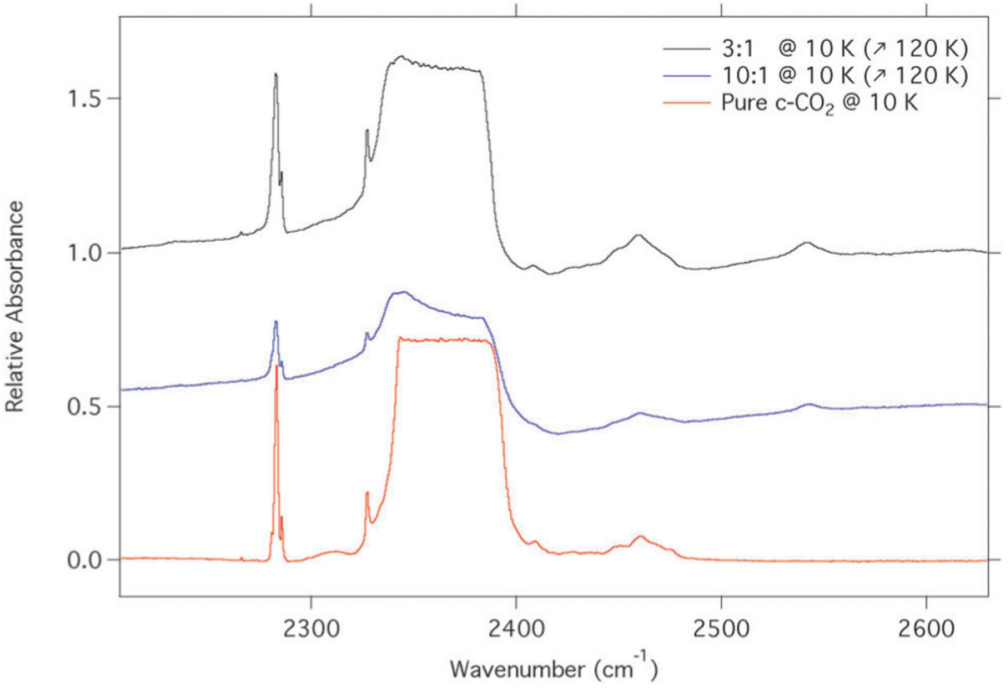

Fig. 7.

FTIR of the $3: 1$ and $10: 1$ mixtures after annealing to $120 \mathrm{~K}$ showing the remaining $\mathrm{CO}_{2}$ in the ice. The transitions are saturated in our spectrometer, and the spectra have been vertically-offset for clarity. A pure c- $\mathrm{CO}_{2}$ spectrum at $10 \mathrm{~K}$ is provided for reference. 


\section{Table 1}

Mixing ratios, total deposition pressure, deposition temperature, substrate temperatures, and annealing temperatures for ices described in this work

\begin{tabular}{lllll}
\hline $\mathbf{C O}_{2}: \mathbf{C H}_{3} \mathbf{O H}$ & $\boldsymbol{P}_{\text {tot }}($ Torr $)$ & $\boldsymbol{T}_{\text {dep }}(\mathbf{K})$ & $\boldsymbol{T}_{\text {sub }}(\mathbf{K})$ & $\boldsymbol{T}_{\text {ann }}(\mathbf{K})$ \\
\hline $1: 3$ & 5.7 & 80 & $10,30,60$ & $90,120,140$ \\
$1: 10$ & 5.4 & 80 & $10,30,60$ & $90,120,140$ \\
$1: 1$ & 5.7 & 80 & $10,30,60$ & $90,120,140$ \\
$3: 1$ & 5.7 & 80 & $10,30,60$ & $90,120,140$ \\
$10: 1$ & 5.7 & 80 & $10,30,60$ & $90,120,140$ \\
$\mathrm{CO}_{2}$ & 2.0 & 80 & $10,30,60$ & $90,120,{ }^{a} 140$ \\
$\mathrm{CH}_{3} \mathrm{OH}$ & 4.0 & 80 & $10,30,60$ & $90,120,140$ \\
\hline
\end{tabular}

${ }^{a}$ After annealing to $120 \mathrm{~K}$, the majority of the $\mathrm{CO}_{2}$ ice had sublimed. After the $140 \mathrm{~K}$ annealing, no $\mathrm{CO}_{2}$ ice remained. 